\title{
DataUSP: Conjunto de serviços analíticos para apoio à tomada de decisões em uma instituição de ensino superior
}

\section{DataUSP: Set of analytical services to support decision making in a higher education institution}

DOI: $10.46814 / l a j d v 3 n 4-056$

Recebimento dos originais: 01/05/2021

Aceitação para publicação: 31/06/2021

\section{Marino Hilario Catarino}

Formação acadêmica mais alta: Mestre em Ciência da Computação - Instituto de Matemática e

Estatística da Universidade de São Paulo (IME-USP)

Instituição de atuação atual: Instituto de Matemática e Estatística da Universidade de São Paulo

(IME-USP)

Endereço completo (pode ser institucional ou pessoal, como preferir): Rua do Matão, 1010, CEP 05508-090 - São Paulo - SP

E-mail: marino@usp.br

\section{Bruno Padilha}

Formação acadêmica mais alta: Mestre em Ciência da Computação - Instituto de Matemática e Estatística da Universidade de São Paulo (IME-USP)

Instituição de atuação atual: Aluno de Doutorado em Ciência da Computação no Instituto de Matemática e Estatística da Universidade de São Paulo (IME-USP).

Endereço completo (pode ser institucional ou pessoal, como preferir): Rua do Matão, 1010, CEP

$$
\text { 05508-090 - São Paulo - SP }
$$

E-mail: brunopadilha@usp.br

\section{João Eduardo Ferreira}

Formação acadêmica mais alta: Professor Titular do Departamento de Computação do Instituto de Matemática e Estatística da Universidade de São Paulo (IME-USP)

Instituição de atuação atual: Departamento de Computação no Instituto de Matemática e Estatística da Universidade de São Paulo (IME-USP)

Endereço completo (pode ser institucional ou pessoal, como preferir): Rua do Matão 1010, CEP

$$
\text { 05508-090 - São Paulo - SP }
$$

E-mail: jef@ime.usp.br

\section{RESUMO}

Este artigo descreve uma aplicação de Business Intelligence para tomada de decisões em uma instituição de ensino superior. A estratégia adotada é descrita considerando as áreas de negócio. O maior diferencial do DataUSP está em caracterizar bem o indicador, que é definido como formas de representação quantificáveis de características de serviços ou processos, utilizado para acompanhar e melhorar os resultados ao longo do tempo.

\section{ABSTRACT}


This paper describes a Business Intelligence Application for decision-making in a higher education institution. The strategy adopted is described considering the business areas. The DataUSP main differential is in the best characterization of the indicator that is defined as quantifiable representation forms of services characteristics or processes, to monitor and improve results over time.

\section{INTRODUÇÃO}

A tomada de decisões em uma instituição de ensino superior envolve não somente o corpo docente e discente vinculado à instituição, como também a responsabilidade social perante a sociedade em que se encontra inserida, o que amplifica sua importância [Calderón 2006]. Ao longo da evolução das instituições de ensino, diversos aplicativos operacionais foram desenvolvidos para atender tanto às necessidades acadêmicas quanto administrativas, como recursos humanos e financeiro. Entretanto, percebe-se a necessidade de elaboração de um aplicativo voltado especificamente à gestão estratégica.

As necessidades operacionais em uma instituição de ensino superior acabam consumindo muitos recursos para serem atendidas e, apesar de existir muitos estudos sobre a gestão em instituições [Godoy 2011], a oferta de aplicativos dedicados à tomada de decisões nas diversas áreas de atuação é restrita. Um Planejamento Estratégico adequado é apontado em diversos trabalhos [Ferreira 2006] como uma solução. Apesar de já existirem no mercado algumas ferramentas de Business Intelligence, como o Tableau (www.tableau.com) e o SpagoBI (www.spagobi.org), a adequação ao ambiente acadêmico é esparsa e a esquematização em um aplicativo é custosa. Isso se deve às diversas características que compõem os indicadores de produtividade de uma instituição de ensino superior.

Tendo o objetivo de suprir essa necessidade, foi desenvolvido o aplicativo DataUSP, que contém diversas ferramentas analíticas para auxiliar na tomada de decisões em uma instituição de ensino superior, tendo como premissa atuar na complexidade das informações de modo a sumarizar os dados mais relevantes a cada área de atuação.

Além das informações existentes nas bases corporativas de uma instituição, o DataUSP dispõe de um conjunto de ferramentas para extração e análise dos dados dos programas de pós-graduação da instituição que são enviados à Capes anualmente. Como resultado, fornece um relatório com histórico do extrato Qualis dos programas e o desempenho de cada docente, permitindo filtrar por períodos específicos.

Outro ponto importante para a análise de uma instituição é a quantidade de trabalhos publicados e o número de vezes que foi citado por outros autores, tanto internamente quanto externos à instituição. As principais fontes que contabilizam o número de citações são o Scopus, Web of Science e o Google Scholar, sendo todas relevantes no meio acadêmico [Kulkarni 2009]. Por meio da quantidade de 
publicações e de suas respectivas citações, define-se uma métrica quantitativa denominada índice-h [Hirsch 2005], que é uma proposta para quantificar a produtividade e o impacto de pesquisas individuais ou em grupos baseando-se nos artigos mais citados.

Todos os serviços do DataUSP apresentam inicialmente um panorama global da instituição, permitindo, por meio de uma navegação simples e intuitiva, iniciar com a visualização da informação sumarizada da instituição de ensino superior, percorrer informações específicas de uma unidade ou departamento e alcançar as informações de um docente. Essa estratégia de navegação permite percorrer da maior (instituição) para a menor (docente) granularidade de um modo ágil, prático e intuitivo.

\section{METODOLOGIA PROPOSTA}

O projeto iniciou-se em 2012 com a modelagem dos dados para a criação de um Data Warehouse. Com o domínio dos dados bem definido, iniciou-se o processo de modelagem do sistema e a escolha das tecnologias que seriam utilizadas.

Uma mesma informação pode ser armazenada em um banco de dados com dois propósitos: ser operacional, atuando com inserção, deleção e atualização das informações, ou ser analítico, tendo como objetivo servir apenas para gerar relatórios e permitir uma análise dos dados.

Inicialmente foi realizado um espelhamento de todas as bases de dados corporativas que atendem cada negócio (graduação, financeiro, recursos humanos entre outros), deste modo mantendo a eficiência dos sistemas operacionais e permitindo que o sistema analítico atue de modo independente, não influenciando na performance do operacional. Essas novas bases espelhadas passaram por uma transformação nos dados que resultou no Data Warehouse, no qual são realizadas todas as atividades analíticas (Figura 1).

Como metodologia, foi adotada a orientação a serviços, sendo o aplicativo dividido em dois grandes componentes: servidor de recursos e interface web para a exibição dos dados. Para facilitar a escalabilidade, portabilidade e a manutenção do sistema, o DataUSP foi implementado com a linguagem de programação Java, seguindo as premissas do REST 2.4 [Mumbaikar 2013] por meio do framework Jersey. O formato JavaScript Object Notation - JSON foi adotado como padrão para a troca de mensagens. Com isso obteve-se uma formatação leve para troca de dados baseada em um subconjunto da linguagem de programação JavaScript. 


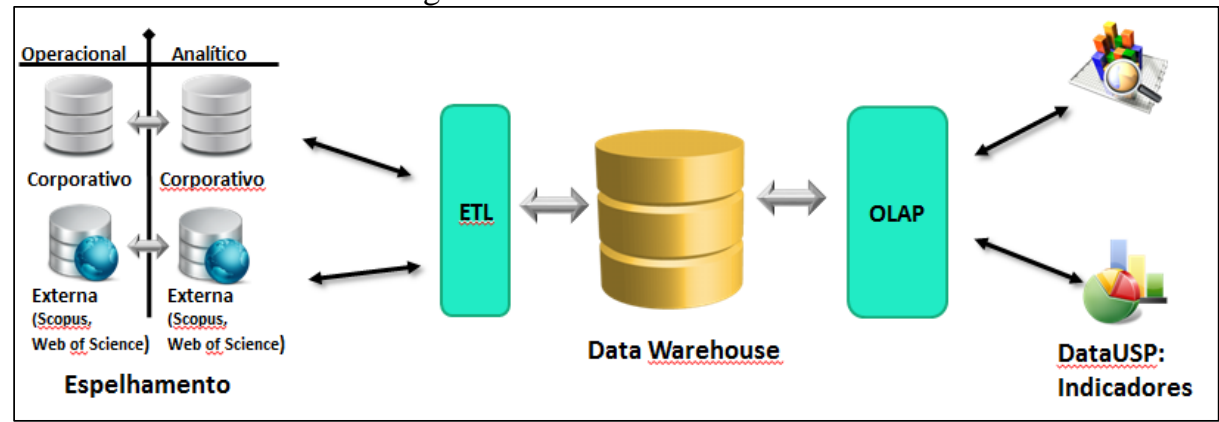

Quanto à visualização dos dados na interface web, foram definidos os seguintes requisitos: exibição de gráficos interativos em três dimensões e renderizados em tempo real; manipulação dos dados com Jquery (http://jquery.com/) e acesso ao servidor por meio de requisições assíncronas AJAX. A biblioteca Fusion Charts (www.fusioncharts.com) foi adotada por atender aos requisitos de integração com JSON e atuar com JavaScript, além de sua diversidade de modelos de gráficos (Figura 2).

Uma vez determinadas as tecnologias a serem utilizadas, iniciou-se o processo de construção de um arcabouço para execução das funcionalidades de Web Services do framework Jersey. O servidor Apache Tomcat, um container Web para executar aplicações que utilizam tecnologias Servlets e JSPs, foi adotado para instanciar-se as classes Java de acordo com as requisições web.

Figura 2. Gráfico das informações com opção de exportação em planilha CSV

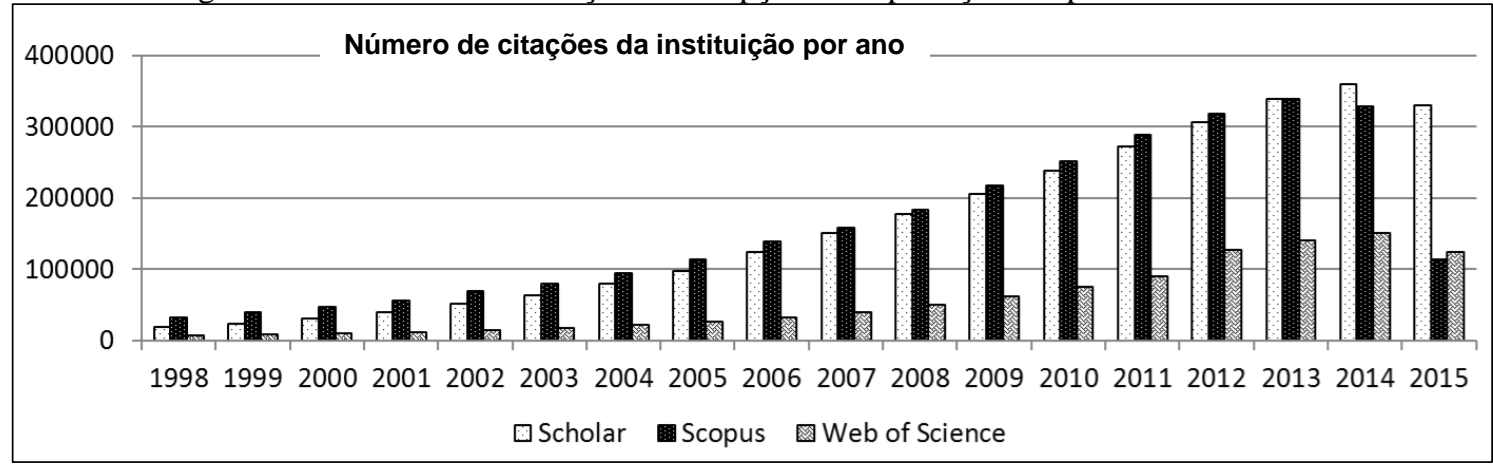

Para receber o retorno do resultado de uma consulta, uma classe Java utiliza o Data Access Object - DAO. Este fornece uma API genérica para acessar dados em diferentes tipos de bancos de dados, executando uma consulta SQL ou uma chamada em uma Procedure e devolvendo o resultado em uma lista de objetos Java conforme cada consulta.

Por fim, temos a classe de negócios, que recebe as requisições e, além de instanciar o DAO, também trata dos dados antes de enviar a resposta para seus métodos. Cada recurso de relatório do 
DataUSP possui essa estrutura, agrupados nas classes de negócio de acordo com sua funcionalidade. As classes de negócio correspondem às áreas de negócio da Universidade: Ensino, Pesquisa e Extensão.

Quanto à visualização, os relatórios exibem os dados na forma de gráficos interativos e também em tabelas, as quais podem ser exportadas como planilhas no formato CSV, para permitir o manuseio dos dados conforme cada necessidade.

Apesar de cada área de negócio dispor de um conjunto próprio de indicadores, a estratégia de identidade visual adotada permite alternar entre as áreas seguindo um padrão uniforme.

\section{CONSIDERAÇÕES FINAIS}

Para uma melhor compreensão da situação atual de uma instituição de ensino, é fundamental realizar uma análise abrangente e precisa dos dados acumulados ao longo dos anos. É nesse contexto que surgiu o DataUSP como conjunto de serviços analíticos da pós-graduação da Universidade de São Paulo para auxílio na tomada de decisão.

Os dados fornecidos pelo DataUSP são essenciais para se conhecer a evolução das diversas áreas de negócio, assim como para detectar as tendências e manter o padrão de excelência dos cursos e programas. Obter e tratar estes dados em tempo real é uma solicitação indispensável para maior agilidade na tomada de decisões e identificação de anomalias que demandem ações estratégicas. A utilização de uma arquitetura orientada a serviços provou-se bastante adequada para o ambiente USP, garantindo alta escalabilidade de recursos computacionais e para simplificar a implementação de novas funcionalidades, facilitando a comunicação com outros futuros Web Services, além de definir um novo paradigma para a construção de sistemas administrativos dentro da universidade. 


\section{REFERÊNCIAS}

Calderón, A. I. (2006) "Responsabilidade Social Universitária: Contribuições para o Fortalecimento do Debate no Brasil", Revista da Associação Brasileira de Mantenedoras de Ensino Superior, Brasília, v.24, n. 36, p. 7-22, jun. 2006.

Ferreira, H. C. C., Ueno, E. M., Kovaleski, J. L. and Francisco, A. C. (2006), "Planejamento Estratégico, Ferramenta Indispensável para Gestão de Instituições de Ensino Superior IES Privadas", Em: III SEGeT - Simpósio de Excelência em Gestão e Tecnologia, Resende, 2006.

Godoy, V. A. and Machado, M. (2011) "Planejamento Estratégico na Gestão Educacional: Uma Ferramenta Importante no Processo Decisório da Instituição de Ensino Superior”, Revista Científica Intraciência, Ano 3, nº 3, p.32-85, Dez 2011.

Hirsch, J. E. (2005). An index to quantify an individual's scientific research output. Proceedings of the National Academy of Sciences, USA, 102(46), 16569-16572.

Kulkarni, A. V., Aziz, B., Shams, I. and Busse, J. W. (2009) "Comparisons of Citations in Web of Science, Scopus, and Google Scholar for Articles Published in General Medical Journals", JAMA The Journal of the American Mecial Association, vol 302, n 10, Setembro de 2009.

Mumbaikar, S., Padiya, P. (2013) "Web Services Based On SOAP and REST Principles", International Journal of Scientific and Research Publications, Volume 3, Issue 5, May 2013.

Valcarenghi, E. V., Müller, I. R. F., Teza, P., Dandolini, G. A. and Souza, J. A. (2012) "Sistemas De Informação Como Ferramenta No Processo De Tomada De Decisão: O Caso Do Hu-Ufsc", Em: XI Congresso Brasileiro de Gestão do Conhecimento, KM Brasil 2012, São Paulo, 2012. 